\title{
Preparation and evaluation of an anti-asthmatic gel of theophylline with diclofenac sodium
}

\begin{abstract}
Asthma is one of the most common diseases worldwide that causes inflammation of airways. Usually different anti-asthmatic drugs are used in asthma treatment through oral route or as nebulizer. In this study we make an effort for formulation and preparation of a transdermal gel using theophylline with Nonsteroidal anti-inflammatory drugs (NSAIDs) diclofenac sodium. Carboxymethylcellulose sodium was used as the gelling material and propylene glycol as penetration enhancer. Different tests were performed to evaluate the quality and performance of prepared gels including the rheology $\&$ drug release behavior of the preparations. The concentration of drug in the gel containing both theophylline and diclofenac sodium was higher than gel containing only theophylline due to synergism and an increase in concentration with time was notified. No significant change on organoleptic properties was found and absence of syneresis indicates good physical stability of gels. Prepared gels showed good and consistent partition co-efficient value for theophylline at different time intervals while diclofenac sodium had lower value than theophylline. Prepared gels give their characteristic pseudoplastic flow with slope value of $>1$ when shear rate is plotted against shear stress on log scale. Release pattern of drug in phosphate buffer ( $\mathrm{pH} 5.5$ ) was studied using a low density polyethylene sheet simulating biological membrane with and without sonication. Moreover, an increase in drug release in 30 minutes with sonication was found. At the same time the release was found to be time dependent.
\end{abstract}

\author{
Volume 8 Issue 2 - 2019
}

\section{Jannatul Fardous, 1,2 Faria Farzana Perveen, ${ }^{3}$ AHM Saifuddin, ${ }^{4}$ Mahmuda Akter, ${ }^{2}$ Sakina Sultana ${ }^{4}$ \\ Department of Chemical Engineering, Kyushu University, Japan 2Department of Pharmacy, Cumilla University, Bangladesh ${ }^{3}$ Department of Pharmacy, Mawlana Bhashani Science \& Technology University, Bangladesh \\ ${ }^{4}$ Department of Pharmacy, Jahangirnagar University, Bangladesh}

Correspondence: Jannatul Fardous, Department of Chemical Engineering, Faculty of Engineering, Graduate School, Kyushu University, 744 Motooka, Nishi-ku, Fukuoka 819-0395 Japan, Department of Pharmacy, Cumilla University, Kotbari, Cumilla-3506, Bangladesh, Tel +8I 8042890083 Email fardousj I I@cou.ac.bd

Received: March 14, 2019 | Published: April 02, 2019

Keywords: asthma, theophylline, diclofenac sodium, gel, sonication

\section{Introduction}

Asthma is defined as a chronic inflammatory disease of the airways and is most common respiratory disease in the whole world. This chronic inflammation is associated with airway hyper-responsiveness like to triggers, such as allergens and exercise. ${ }^{1}$ This is followed by constriction of bronchial smooth muscles, hyper secretion of mucus that leads to narrowing of airways. ${ }^{2}$ As a result patients suffer from the characteristic symptoms of asthma like breathlessness, chest tightness, wheezing etc. particularly in night or early in the morning (Figure 1). ${ }^{3,4}$

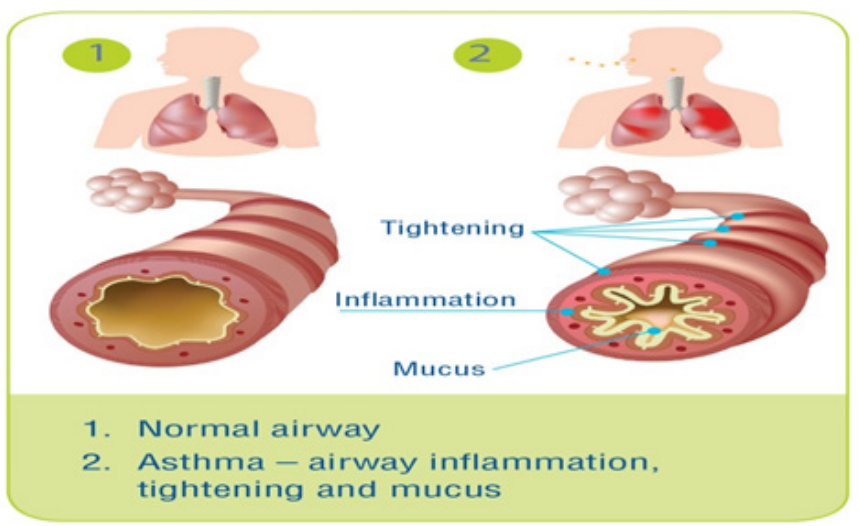

Figure I Difference between normal airways and asthmatic airways. ${ }^{4}$

The risk factors that stimulate asthma attacks may vary from person to person. Usually allergen, environmental factors, food habits trigger asthma attack. ${ }^{5}$ Family history of asthma increases the possibility and occurrence of asthma in a person. ${ }^{3}$ Despite significant improvements in the diagnosis and management of asthma over the past decade, as well as the availability of comprehensive and widelyaccepted national and international clinical practice guidelines for the disease, asthma control in still now remains suboptimal. ${ }^{1}$ Asthma kills around 1000 people every day and affects as many as 339 million people worldwide (The global asthma report 2018). According to WHO, asthma is a most common disease among children also on the elderly. ${ }^{6}$ Over the last 40 years, there has been a sharp increase in the global prevalence, morbidity, mortality and economic burden associated with asthma and rates are increasing in low and middle income countries mainly due to westernization. ${ }^{7}$ Now-a-days asthma appears to be a substantial public health problem in Bangladesh. About 11.6 million people including 4.1 million children suffer from asthma-related symptoms. ${ }^{5}$ Asthma is creating a substantial burden to individuals and families and possibly restricting individuals' activities for a lifetime as it is an under-diagnosed and under-treated disease till now. ${ }^{6}$

Different types of bronchodilators and steroids are used in the treatment of asthma for decades. Theophylline is one of the bronchodilators belongs to methylxanthin group and widely used in the treatment of chronic asthma. This drug has been using over the last 50 years or so. ${ }^{8}$ Sometimes theophylline is administered with glucocorticosteroids for preventing nocturnal asthma attack. On long term use steroidal drugs causes many side effects mainly immunosuppression, osteoporosis, Cushing's syndrome, fatigue, high blood sugar and so on. ${ }^{9}$ Currently available anti-asthmatic preparations are administered per oral or through nebulization. These routes of administration sometimes cause poor patient compliance in children and elder patients. ${ }^{10}$ Similarly most of oral preparations are solid in 
nature and contain glycogenic materials like sorbitol, glycerin etc. which are unsuitable for diabetic patients. ${ }^{11}$ Transdermal route became very popular in recent days as they increase drug bioavailability as well as patient compliance. ${ }^{12} \mathrm{Gel}$ is a semisolid dosage form with three dimensional crosslinking structure applied topically on the skin. Depending on the site of action gel can induce either local or systemic effect. ${ }^{13}$ Among different types of gels hydrogel is used widely in drug delivery system. There high content of water make them more biocompatible. At the same time hydrogel has the ability to determine the changes on $\mathrm{pH}$, temperature, concentration of metabolite and cope with such changes which make it an ideal delivery system. ${ }^{14}$

Though gel offers certain major advantages over other dosage forms, transdermal preparation (gel) containing theophylline is not available till today in Bangladesh. Therefore our study aimed at the formulation and preparation of theophylline (TH) with diclofenac sodium (DS) in hydrogel material carboxymethylcellulose sodium (CMC-Na). DS is a nonsteroidal anti-inflammatory drug (NSAID) and gives anti-inflammatory action by reducing prostaglandin release from cyclooxygenase (COX). Some evidence indicates it inhibits the lipoxygenase pathways, thus reducing formation of the leukotrienes which damage the epithelium and cause bronchial hyper-responsiveness. ${ }^{15}$ So this drug can be administered along with theophylline to reduce airways inflammation. Stability study and different physicochemical properties of gel were tested to determine the feasibility of TH gel with DS which may be a new approach for asthma treatment.

\section{Materials and methods}

\section{Materials}

Theophylline anhydrous (TH) and Diclofenac sodium (DS) were gift from Square Pharmaceuticals Ltd, Carboxymethylcellulose sodium (CMC-Na), Propylene glycol (PG), Methyl paraben, Distilled water, Methanol were gifts from Department of Pharmacy, Jahangirnagar University.

\section{Methods}

Preparation of gel: According to the method described by Loyd, CMC-Na was dissolved in water and PG solution using magnetic stirring. ${ }^{16}$ It takes about 30 min to completely dissolve. A smooth gel is formed which was then sonicated in water bath sonicator (Decon Ultrasound, England) for 10 minutes to remove entrapped air. A solvent system of methanol and water in 3:1 ratio was prepared and both $\mathrm{TH}$ and DS drugs are dissolved in it separately. Drug concentration was $1 \% \mathrm{w} / \mathrm{w}$ of gel for both drugs individually and solution of dissolved drugs was then added with gel base. After proper mixing a transparent gel is formed containing a bronchodilator and a NSAID. The same was followed to prepare a gel of same concentration using the drug theophylline only in order to compare the effect of theophylline in different preparations. The gels were kept in glass containers and different physicochemical properties were performed periodically. The whole procedure is graphically presented in Figure 2.

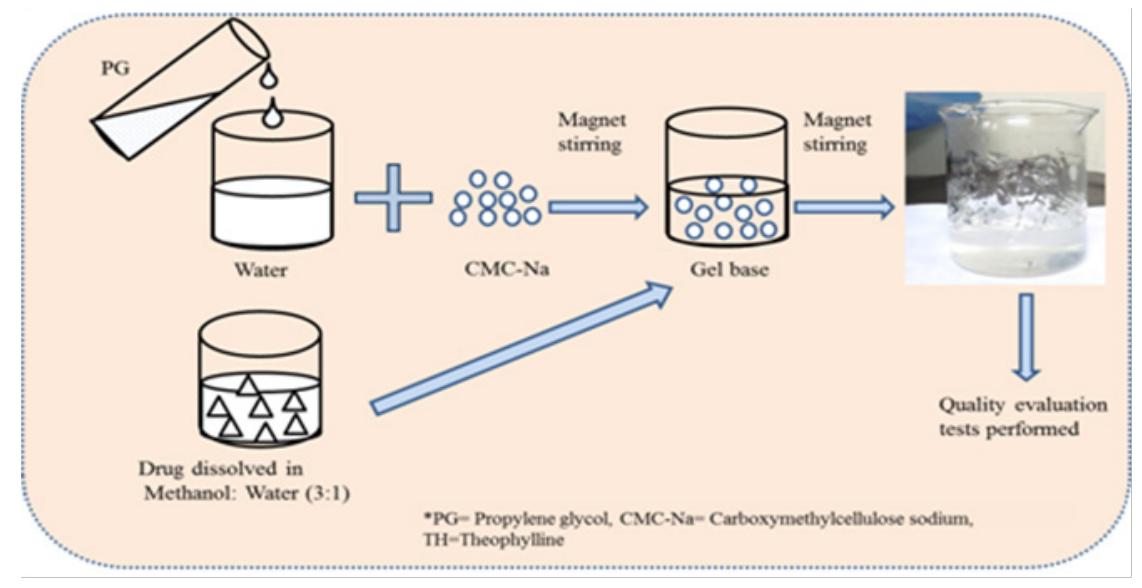

Figure 2 Graphical presentation of theophylline gel preparation procedure.

Organoleptic properties: Different organoleptic properties namely color, odor, texture were observed visually during the experimental time period of two months. Quality marks on these properties were given by human volunteers of age 20-25 years according to the typical thermometer scale of consumer acceptability.

Syneresis: Gel synersis was observed by keeping $2 \mathrm{gm}$ of gel in room temperature during the storage period. The gel was observed visually for any kind of drying out or separation of liquid phase.

Potency: Drug potency was determined by mixing $1 \mathrm{gm}$ of gel with water followed by filtration and dilution. Then absorbance of filtered solution was determined using UV-visible spectrophotometer (Shimadzu 1601 PC, Japan) at wavelength of $214 \mathrm{~nm} \& 200 \mathrm{~nm}$ for theophylline and diclofenac sodium respectively. The concentration of drug for each gel preparations was determined from standard calibration curve drawn with absorbance versus six concentration values ranging from $1 \mu \mathrm{g} / \mathrm{ml}$ to $20 \mu \mathrm{g} / \mathrm{ml}$.
Partition co-efficient: A definite quantity of gel was mildly shaken with water and chloroform in a separating funnel to determine the partition coefficient. After evaporation of solvent followed by dilution, drug concentration in water and organic solvent were determined from absorbance.

Rheology: For rheology 1 gm of gel was taken and spreaded on a glass slide to cover an area of $1 \mathrm{~cm} \mathrm{X} 1 \mathrm{~cm} \mathrm{X} 0.1 \mathrm{~cm}$. A cover slip was placed over the gel and weight was applied gradually on it until the gel collapsed. Changes on area of gel with applied stress were noted. Then the rate of shear $(\mathrm{G})$ was plotted against shearing stress $(\mathrm{F})$ on log scale and flow pattern (rheology) of gel was determined.

Effect of sonication on drug release rate: To obtain the release rate of drug from the gel preparations, $2 \mathrm{gm}$ of each gel was taken and spreaded on low density polyethylene sheet which mimics the human skin and then tied on the open end of test tubes. Each test tube was then dipped on separate beakers containing PBS of pH 5.5 and placed 
on a water bath sonicator. $1 \mathrm{ml}$ of aliquot was withdrawn and replaced with fresh medium simultaneously from the beaker at different time intervals for 30 minutes. Absorbance was taken at corresponding wavelengths after dilution with PBS to determine drug release rate. The same was followed for both of the gel in the absence of sonication also.

\section{Results}

In the present study different properties of gel were investigated to evaluate the quality of the preparation. Results have been shown in different figures and in table.

\section{Organoleptic properties \& syneresis}

Results based on visual inspection of color, odor, texture and syneresis which indicate physical stability of gel are given on Table 1.

Table I Organoleptic properties and syneresis results of prepared gels

\begin{tabular}{lllll}
\hline Day & Color & Odor & Texture & Syneresis \\
\hline 1 & Colorless & Good & Smooth & Absent \\
7 & Colorless & Good & Smooth & Absent \\
15 & Colorless & Good & Smooth & Absent \\
30 & Colorless & Good & Smooth & Absent \\
60 & Colorless & Good & Smooth & Absent \\
\hline
\end{tabular}

\section{Potency}

Concentration of TH and DS in the gel during experimental time period was determined. Higher concentration of TH was present in gel containing TH and DS than the gel containing TH only (Figure $3)$. This indicates the synergistic effect of DS on TH distribution on vehicle.

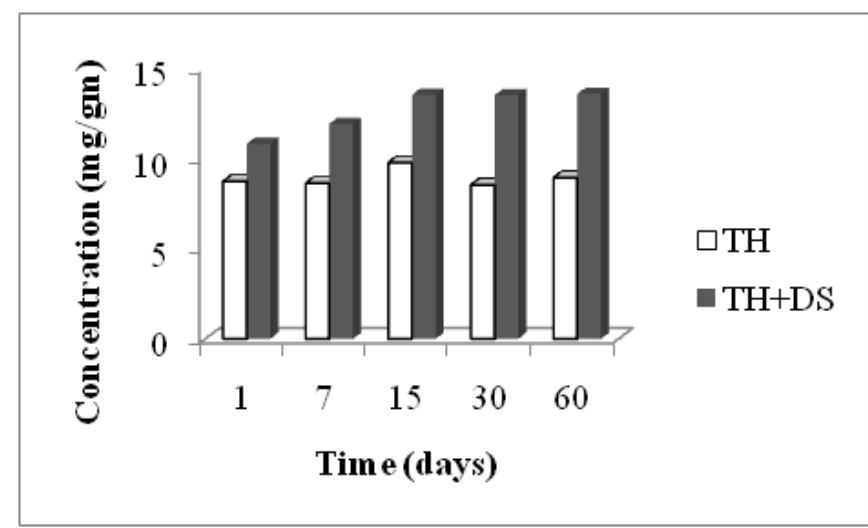

Figure 3 Concentration of theophylline in different gel preparations.

\section{Partition co-efficient}

Partition co-efficient $(\mathrm{P})$ results shows that the value of $\mathrm{P}$ was $\approx 0.5$ for theophylline in both TH and TH+DS gel at 15 minutes, 30 minutes and 45 minutes (Table 2). In case of diclofenac sodium $\mathrm{P}$ value was lower than $\mathrm{TH}$ due to its hydrophilic nature.

\section{Rheology}

Plotting shearing rate against shearing stress in $\log$ scale, exponential term of the plot were found to be 1.5 and 1.4 for theophylline gel and gel containing both TH and DS respectively (Figure 4).

Table 2 Partition co-efficient of different gel preparations at particular time in-vitro

\begin{tabular}{llll}
\hline \multirow{2}{*}{ Time (min) } & TH gel & \multicolumn{2}{c}{ TH + DS gel } \\
\cline { 3 - 4 } & & DS & TH \\
\hline 15 & 0.53 & 0.2 & 0.49 \\
30 & 0.58 & 0.47 & 0.52 \\
45 & 0.56 & 0.37 & 0.42 \\
\hline
\end{tabular}
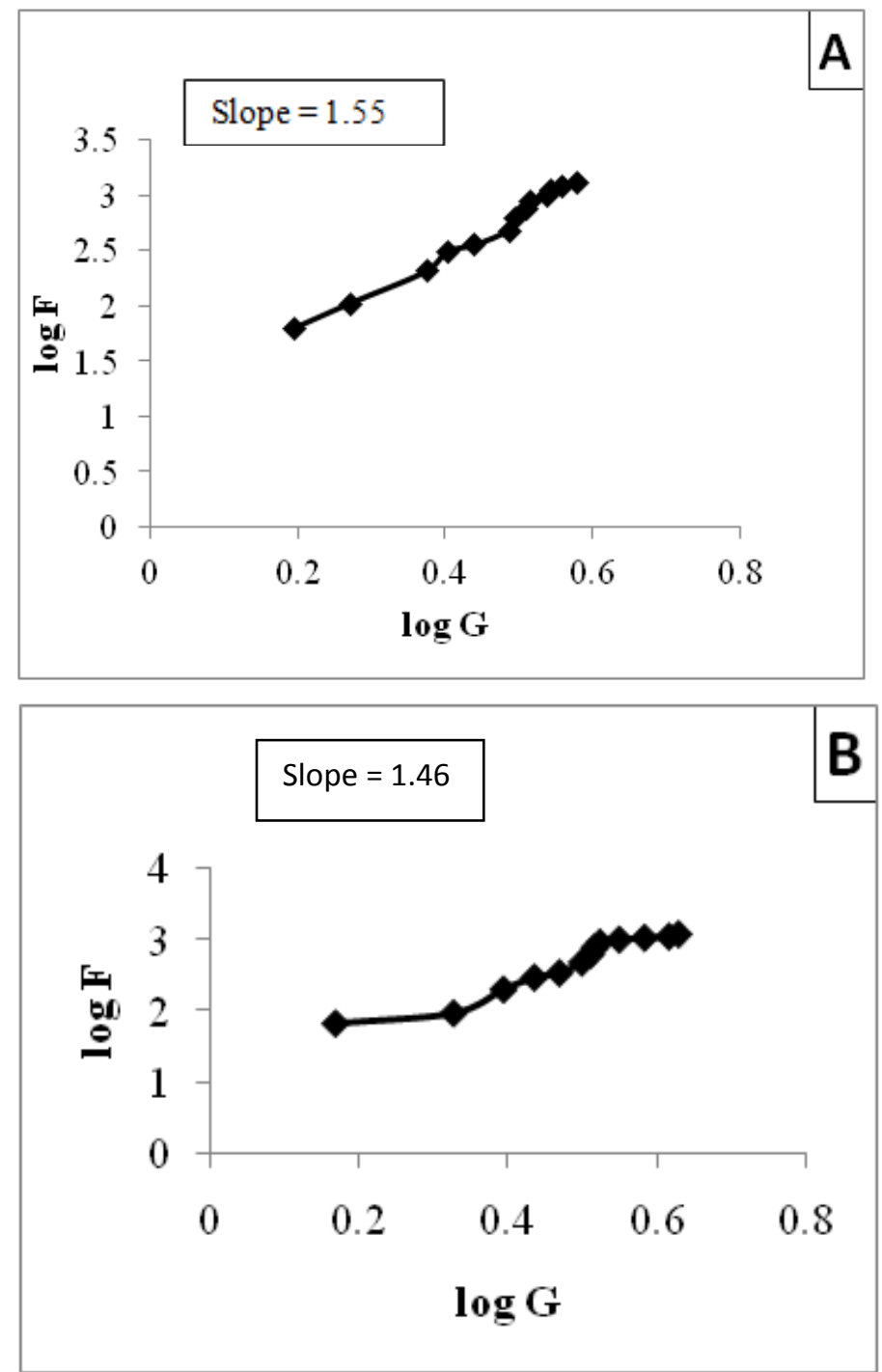

Figure 4 Rheogram of Theophylline gel (A) \& Theophylline \& Diclofenac sodium gel (B).

\section{Effect of sonication on drug release}

One of the interesting finding of our study is the effect of sonication on drug release. Results exhibited an increase in drug release upon sonication (Figure 5). 


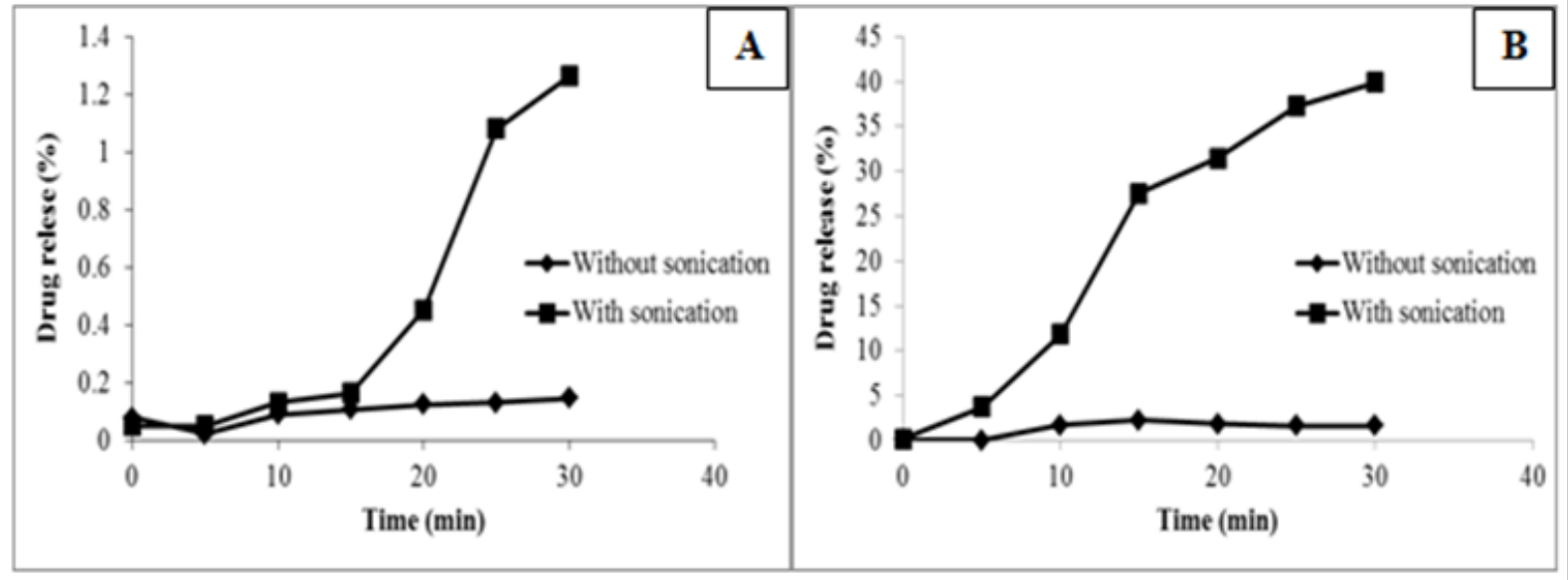

Figure 5 Comparison of drug release rate from Theophylline gel (A) \& Theophylline \& Diclofenac sodium gel (B) with and without sonication respectively.

\section{Discussion}

Results of different physicochemical properties of our prepared gels are found quite well. The aesthetic properties were found acceptable during the experiment. At the same time no syneresis as well as drying out of gels takes place. These indicate a good quality and physical stability of theophylline gel both alone and with diclofenac sodium. In case of potency, TH concentration was found higher for gel containing both TH and DS than gel with TH alone. This may be synergistic effect of one drug to another which causes increased solubility and uniform distribution of TH throughout the gel. There was a change in drug concentration with time but was not significant. One of the crucial criteria for transdermal preparation is its partition co-efficient $(\mathrm{P})$ that was also tested in our research. $\mathrm{P}$ value for theophylline in all the preparation was 0.5 which ensures better permission tendency through the hydrophobic layer of skin. The values were consistent with time at room temperature that ensures a crucial criterion of partitioning i.e. partition co-efficient must be independent of time and temperature. On the other hand, a significant fall in $\mathrm{P}$ values for diclofenac sodium was found. This may occur due to effect of theophylline on partitioning of diclofenac sodium. However, the partition co-efficient of a drug between the membrane and solvent mixture generally falls as the solubility in the system rises. Hence it is important not to oversolubilize a drug in order to promote penetration and the formulation should be at or near saturation. ${ }^{17}$ Sometimes theophylline is used to increase the solubility of other drugs as a hydrotropic agent. Thus the decrease in the magnitude of partition co-efficient for diclofenac sodium in gel enclosing both TH and DS may be due to the over soubilization of the drugs. Further studies are needed in this regard to ensure the effect of theophylline as a hydrotropic agent. Rheology study of gel showed that the gel are non-newtonian system and have pseudoplastic flow as the slope value was $>1$ for all the preparations. According to Lachman et al., ${ }^{18}$ it is claimed that if the power term of curve plotted $\log \mathrm{G}$ vs $\log \mathrm{F}$ is $>1$, the flow would be pseudoplastic. So our findings are in line with them. Last of all we found significant effect of sonication on drug release. Increase in drug release due to sonication was found for both of the gel. If the effect of sonication is considered, the release of theophylline from the TH+DS gel was found much higher than gel with TH alone. For theophylline gel, $\approx 1 \%$ drug was released in 30 minutes in the absence of sonication while it was $1.26 \%$ with sonication at the same time course Effect of sonication was much higher in case of gel containing both $\mathrm{TH}$ and DS. Drug release was $1.6 \%$ and $\approx 40 \%$ in 30 minutes in the absence and presence of sonication respectively. Such base line lifting for the
$\mathrm{TH}+\mathrm{DS}$ gel might have been taken place as the wavelength value for diclofenac sodium had a sharp difference of $14 \mathrm{~nm}$ then theophylline. Thus our study established that sonication enhanced drug release from gel and release is time dependent.

\section{Conclusion}

Our study has shown promising data for gel rheology and drug release as well as potency of drug. At the same time data showing effect of sonication regarding amount of drug release across the microfilm barrier also encouraging. Therefore it can be concluded that it is possible to formulate and prepare 'gel' containing an antiasthma drug along with an NSAID and data can be simulated to in-vivo study for further progression of research.

\section{Acknowledgment}

Authors are grateful to Department of Pharmacy, Jahangirnagar University, Bangladesh for providing support to conduct this work. We are also thankful to Square Pharmaceuticals Ltd, Bangladesh for providing Theophylline \& Diclofenac sodium.

\section{Conflicts of interest}

The authors declare that there is no conflict of interest.

\section{References}

1. H Kim, J Mazza. Asthma. Journal of Allergy, Asthma and Clinical Immunology. 2011;7(1).

2. Expert Panel Report 3: Guidelines for the Diagnosis and Management of Asthma, National Asthma Education and Prevention Program, Third Expert Panel on the Diagnosis and Management of Asthma. Bethesda (MD): National Heart, Lung, and Blood Institute: USA; 2007.

3. RJ Greene, ND Harris. Pathology and Therapeutics for Pharmacists -A basis for clinical pharmacy practice. $3^{\text {rd }}$ ed. 2008:292-325.

4. Asthama. World Health Organization; 2019.

5. MM Mamun, ASA Salauddin, MF Hossain, et al. Prevalence of asthma and its associated factors among the undergraduate students of Bangladesh Agricultural University. International Journal of Natural and Social Sciences. 2016;3(1):32-36.

6. MI Hossain, M Parvez, FT Zohora, et al. Prevalence of asthmatic respiratory complications among the rural community of Tangail area in Bangladesh. International Journal of Community Medicine and Public Health. 2018;5(4):1291-1297. 
7. https://www.lung.ca/sites/default/files/Asthma_airway_E.jpg

8. HP Rang, MM Dale, JM Ritter, et al. Rang and Dale's Pharmacology. $6^{\text {th }}$ ed. 2009:356-366.

9. Y Marandi, N Farahi, GS Hashjin. Asthma: beyond corticosteroid treatment. Arch Med Sci. 2010;9(3):521-526.

10. LL Brunton, JS Lazo, KL Parker. Goodman \& Gilman's The Pharmacological basis of Therapeutics. $11^{\text {th }}$ ed. McGRAW- HILL: Medical Publishing Division: 1941:727-730.

11. RU Shahidi. Quick Index of Medical Products and Problems. 2007:74 75 .

12. D Zaghi, H I Maibach. Survey of Safety and efficacy information in drug inserts for topical prescription medications. Am J Clin Dermatol. 2007;8(1):43-46.
13. LV Allen jr, NG Popovich, HC. Ansel, Ansel's Pharmaceutical Dosage Forms and Drug Delivery Systems. $8^{\text {th }}$ ed. Philadelphia: Lippincott Williams \& Wilkins; 2005:278-279.

14. https://en.wikipedia.org/wiki/Gel

15. DW Scholer, EC Ku, I Boettcher, et al. Pharmacology of diclofenac sodium. Am J Med. 1986;80(4 Suppl 2):34-38.

16. LV Allen, Current and practical compounding information for the pharmacists. Compounding for Phonophoresis. Secundum Artem. 2005;2:748-999.

17. ME Aulton. Aulton's Pharmaceutics: The design and Manufacture of Medicines. $3^{\text {rd }}$ ed. UK: Churchill Livingstone Elseiver; 2007.

18. L Lachman, HA Lieberman, JL Kanig. The Theory and Practice of Industrial Pharmacy. $3^{\text {rd }}$ ed. India: Varghese Publishing house; 2010. 